\title{
Third Generation (3G) Mobile Network Planning Process and Methodology - Case Study
}

\author{
Saed Tarapiah \\ Telecommunication Engineering Dept. \\ An-Najah National University \\ Nablus, Palestine
}

\author{
Kahtan Aziz \\ College of Engineering Computing. \\ Al Ghurair University \\ Dubai, United Arab Emirates
}

\author{
Shadi Atalla \\ College of Information Technology (CIT) \\ University of Dubai \\ Dubai, United Arab Emirates
}

\begin{abstract}
Upgrading from Second Generation (2G) to Third Generation (3G) mobile networks is an important concept for every practicing telecommunications engineer and technical decision maker involved in $3 \mathrm{G}$ planning. Coverage and capacity are important issues in the planning process for cellular $3 \mathrm{G}$ mobile networks. The planning process aims to allow the maximum number of users sending and receiving adequate signal strength in a cell. In this paper we show and apply the methodology and the planning steps for designing a $3 \mathrm{G}$ network on top of existing $2 \mathrm{G}$ mobile network. The roadmap of this paper works, is to define the geographical region under study and as our design target, then, forecasting the subscriber profile is considered and well defined to meet the design parameter for the traffic demand, taking into consideration both current profile and the forecasted future service demand, later, the paper show how to perform both capacity and coverage dimensioning process in order to achieve nominal cell palling.
\end{abstract}

\section{Keywords}

3G, Network Planning, Mobile Communication, Radio Network

\section{INTRODUCTION}

Cellular technology evolution has been started since 1950s, and the first commercial systems came in the late 1970s. Where cellular networks can be classified into different generations, namely, First Generation, Second Generation [11], Third Generation [4] and Fourth Generation [9]. In this paper we study the planning phases in order to upgrade the mobile operator network, to build a third generation in parallel with the current deployed second generation network. Many earlier works have been conducted regarding the $3 \mathrm{G}$ radio network planning i.e. [3], our study is related to the $3 \mathrm{G}$ planning and implementation for Nablus city, as one of the major Palestinian cities. The planning process have some main attributes and factors such as subscriber profile forecasting and the calculation of the service traffic demand, in addition to the capacity coverage requirements. This paper ends up with a proposed for an applicable $3 \mathrm{G}$ network to be deployed in the selected cities.

\subsection{Motivation for Carrying Out this Research}

It is worth to know that the Palestinian cellular network operators are still working on GSM/GPRS/EDGE (Global System for Mo-

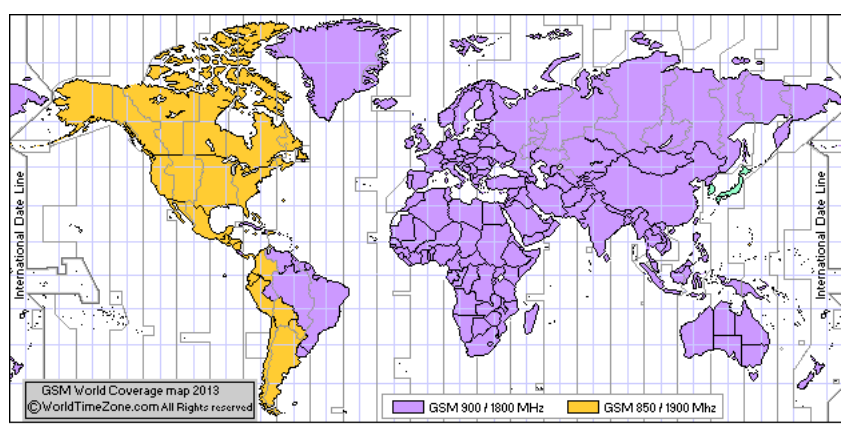

Fig. 1: GSM World Coverage Map 2015.

bile Communications/ General Packet Radio Service/ Enhanced Data Rates of GSM Evolution) that offers voice service and non matured data services. Nowadays, Palestinian subscribers are demanding more data communication services and aligned with the international subscribers demand. As figure 1 represents that GSM is already covered almost all over the world.

As a result, we selected this topic to be aligned with the coming data evaluation in Palestine. This study will be very useful for the current operators to upgrade for the $3 \mathrm{G}$ network.

\section{LITERATURE REVIEW AND RELATED WORK}

In this paper, we outline the "3G Radio Planning Design" in order to plan and design UMTS/3G (Universal Mobile Telecommunications System) network in Palestine. It provides design requirements and assumptions (e.g. service quality, link budgets, etc) that should be used for planning a UMTS network; the Radio network design process is outlined keeping in mind that Radio design will be an overlay on the existing $2.75 \mathrm{G} \mathrm{GSM}$ network. Palestinian telecommunication mobile operators upon getting the approval and the permission to implement the $3 \mathrm{G}$ mobile technology, they have to design and deploy a UMTS network across all regions that will be capable of providing voice and packet data (including High Speed Packet Access HSPA) services to meet the rapidly data demand. The design will be an overlay on the existing GSM networks in Palestine to keep the current available infrastructure for the operational companies and to keep the utilization of current $2.75 \mathrm{G}$ services. 


\subsection{Related Work}

The first case study of $3 \mathrm{G}$ network in Europe, including the design and implementation was undertaken in Isle of Man, as the Manx Telecom $3 \mathrm{G}$ project [17], where all design and planning decisions were based on consideration of the desired end user experience, considering network quality, service coverage and performance of new data applications. Moreover, many other related works have been carried to study the 3G mobile network planning, where Guo et al., 2003 in [5] have studied the coverage and capacity calculations for $3 \mathrm{G}$ mobile network planning, where The planning process aims to allow the maximum number of users sending and receiving adequate signal strength in a cell. Moreover, the work carried in [1] [15] has shown that the network planning process does not depend only on the signal prediction, moreover, it is not appropriate to depends on the classical second system generation in terms of formulas and parameters. Notwithstanding, mathematical programming models for supporting the decisions on where to install new base stations and how to select their configuration (antenna height and tilt, sector orientations, maximum emission power, pilot signal, etc.) are discussed in [1] [14] which finds a trade-off between minimizing costs as well maximizing the covered area. In general the model take into consideration signal-quality constraints and requirements in both directions uplink and downlink, in addition to the power control mechanism and the pilot signal. More sophisticated work has been run, to automate the cellular planning process of the $3 \mathrm{G}$ network as being stated in [13] [16], some more important factors can be taking into consideration during the planning process, besides the coverage plan, and capacity pan, the Quality of Service QoS, resource utilization, and economical aspects have been considered in the work in [17]. In this work, we are going to state and describe full and complete methodology, design steps, and calculations for mobile planning of $3 \mathrm{G}$ network, on top of existing $2 \mathrm{G}$ network, for a given Palestinian city as a case study.

\section{RESEARCH METHODOLOGY}

This paper work has been conducted with the cooperation with Wataniya Mobile [12], The paper work focuses on and discussing three major aspects of radio network planning and design which are: coverage, capacity, and Quality of Service (QoS). The designed methodology is requiring an advanced tools and procedure to accomplish the mentioned parties of the planning. Wataniya Mobile offers the radio network planning tools to be used in their Head Quarter (HQ) and to support the idea and the techniques of capacity design and calculation, the QoS in the WCDMA [7] technology directly linked to the coverage and capacity design. To perform a complete $3 \mathrm{G}$ radio network design, we configured the radio planning tools to meet paper target and scope, the work include filling and configuring $3 \mathrm{G}$ radio capacity sheet to calculate and automate the capacity planning, moreover, to be more close to the actual design which started with nominal design, we did an actual site survey in addition to visit some selected sites of the designed ones based on the radio planning guidelines i.e. to match between the nominal and the actual sites location. At the end, we formulate the design based on the mentioned required important steps which include the coverage, capacity, and QoS steps. This will include the sub-actions like the propagation model, link budget calculations,... etc.

\section{DESIGN PROCEDURE AND ANALYSIS}

ubsectionRadio Network Design Process

The process of designing the radio network is considered as one of the most important and crucial issue in the wireless design since

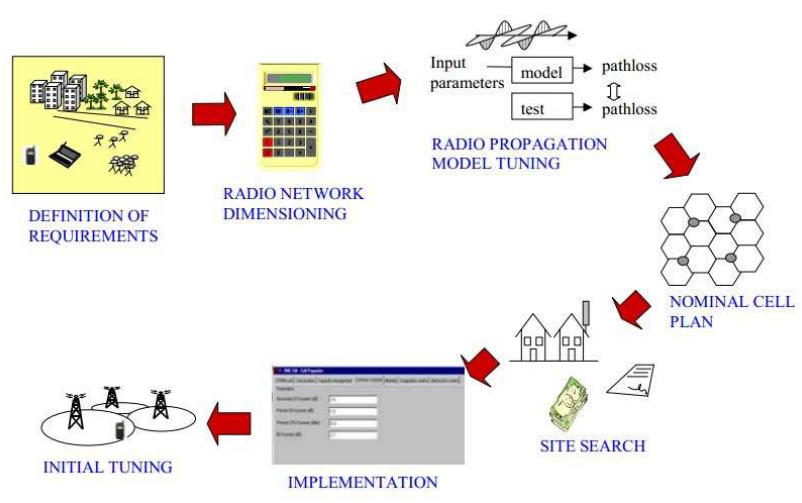

Fig. 2: Radio Network Design Process.

it depends on many variables related to the land terrain, population density, allocated spectrum, and the target itself. The design process for any wireless system could have some common steps like the check list matrix, where figure 2 states the simple flow that will be followed during the planning and design for the $3 \mathrm{G}$ network, the design process can be enumerated as:

(1) Definition of the Requirements: The first stage of the design process is to define the target required of the design, this stage holds surveying for an optimal solution and identifying the required tools and data to in order to start the designing and planning task i.e coverage percentage, forecasted number subscribers, subscriber traffic profile, ...e.t.c.

(2) Radio Network Dimensioning: The next step, involves starting data mining, and to calculate the required capacity and equipments and tolls to meet the forecasted demand.

(3) Radio Propagation Model Tuning: This stage concerns looking for the most important service Key Performance Indicators KPIs which is the coverage footprint, where the WCDMA coverage prediction depends on the loaded traffic, we have to allocate the mentioned step two traffic per sector to the tool in order to have better accuracy in the coverage prediction. The propagation model is one of the most important steps to be tuned and suitable for the land terrain to have the efficient coverage prediction.

(4) Nominal Cell Planning: The planning tool is used to create a nominal cell plan by using the engineering sense and the tools features. The tools will support the engineering decision with many plots analysis related to the coverage, spectrum, and interference.

(5) Site Survey: The cell planner, and site hunters, identify the suitable and applicable sites location that fits the radio coverage requirements. The site leasing/rental issues and the construction obstacles are also taken into consideration at this stage.

(6) Implementation: This step includes all the sub-steps that are required for nominal cell planning. Whereas, planning tools could be used to evaluate many related parameters such as cell parameters, handover cell candidates, as well as the best location from Site Survey step,Antenna, RBS type, feeders,...etc are to be selected in this phase.

(7) Initial Tuning: This step considers performing the drive test of the selected target area, where there are some available drive tests tools which can be applied in this stage, the outcomes and 


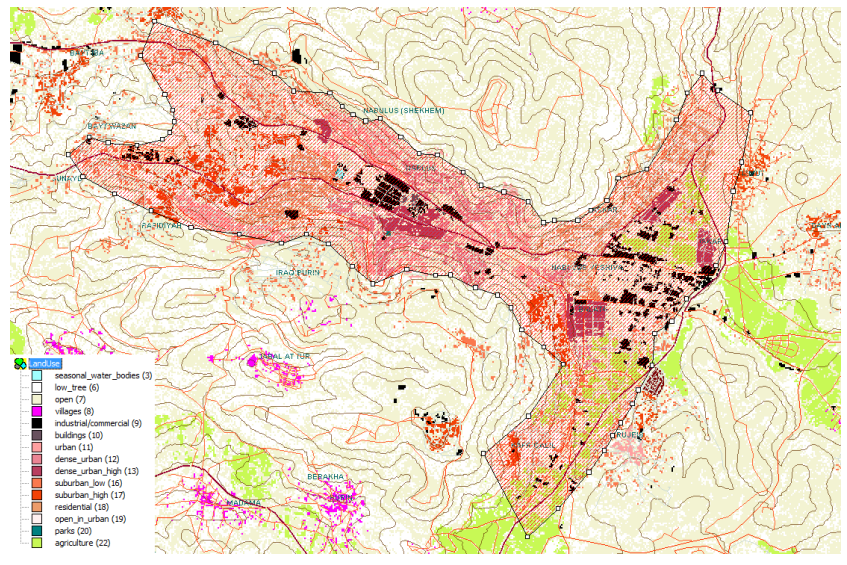

Fig. 3: Nablus city in TEMS digital map.

findings of the related target area measurements will be used further in order to tune the network to achieve the intended KPIs based on the design requirements.

\subsection{Requirements Definition}

The design requirements of the radio network include coverage, capacity and QoS, those requirements are related to different area types: dense urban, urban, suburban and rural during the design assumptions. In addition to these main area types, roads have to be considered due to their importance in the service continuity and traffic volume.

4.1.1 Area and Population. Most WCDMA networks are rolled out in phases, where for each phase of the network the number of km-square of each area classification (Dense Urban, urban, suburban, and rural and road) in addition to the subscribers distribution per area have to be defined and determined. Starting with Nablus city, and by using the digitized map that is typically supported by any planning tools (TEMS [8] Cell planner are used in this projectit is a Swedish tool). The used digital map is created from high resolution satellite in 2015 and have 2 meter resolution which can be described as an accurate digital map, the used map have Palestinian land terrain, elevations, land use (urban, sub urban etc) and vectors (roads, main streets,...), figure 3 shows an example of Nablus city based on the available and the digital map in use for the land use purposes.

As per the digital map filtering, the area to be used for the planning issues will be $20.57 \mathrm{Km}$-square which could be classified as mentioned in the digital as stated in table 1

According to statistics of the Palestinian Central Bureau of Statistics in 2015 , Nablus city has 187,839 people, so we expect that the number of population in 2018 to be around 205,000 people based on the population growth in west-bank/Palestine. The design assumption is targeting to serves $30 \%$ of the population as a market share which means we will end up with 60,000 subscribers at the end of 2018 .

4.1.2 Required Equipments. This section enumerates the required equipments for the implementation of the proposed $3 \mathrm{G}$ network, as being described:

(1) Radio Base Station (RBS) Main remote Solution A Main Remote solution, optimized to deliver high radio performance for efficient cell planning in a wide range of indoor and out-

\begin{tabular}{|c|c|}
\hline Category & In Km-Square \\
\hline Seasonal water bodies & 0.01 \\
\hline Low tree & 3.79 \\
\hline Open & 9.23 \\
\hline Industrial/commercial & 0.98 \\
\hline Buildings & 0.02 \\
\hline Urban & 1.03 \\
\hline Dense urban & 0.92 \\
\hline Dense urban high & 0.68 \\
\hline Suburban low & 0.8 \\
\hline Suburban high & 0.76 \\
\hline Residential & 1.11 \\
\hline Open in urban & 0.02 \\
\hline Parks & 0.01 \\
\hline Agriculture & 1.19 \\
\hline
\end{tabular}

Table 1. : Nablus city areas classification

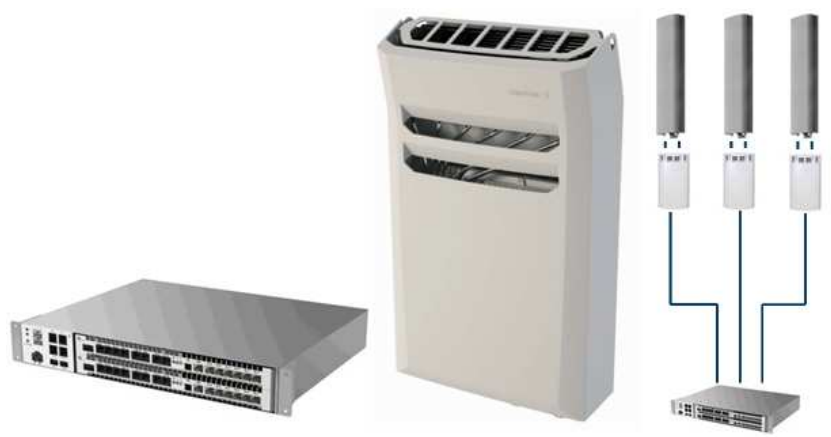

Fig. 4: Ericsson RBS MU, RRU and 3-sector site respectively.

door applications. The Main Remote Radio Base Station, in which each Remote Radio Unit (RRU) is located near an antenna, reduces feeder losses and enables the system to use the same high-performance network features at lower output power, thereby lowering power consumption and both capital and operational expenditure. The Main Remote concept is designed to support all technologies in virtually any combination. The main Remote Solution is divided into a Main Unit (MU) and multiple Remote Radio Unit (RRU) that are connected to the MU through optical fiber cables. Where figure 4 shows RBS MU, Remote Radio Unit RRU and RBS 3-sector site respectively.

(2) Remote Radio Unit Remote Radio Unit WCDMA (RRUW) and Remote Radio Unit Standard (RRUS) are designed to be installed close to the antennas, and can be either wall or pole mounted. The RRUW has got WCDMA capability, and it is Multi Standard Radio (MSR) capable, that means RRUS is capable of running GSM, WCDMA and LTE on the same RRU hardware. The unit configuration can be done by software reload. The RRUS hardware is prepared for running mixed mode configurations, where RRUW and RRUS sustainable average output power is 60 Watt, for very large coverage and high capacity requirements. Dual band configurations are also supported by connecting RRUW or RRUS for different frequency bands to the same MU. The RRUW and RRUS contain most 


\begin{tabular}{|c|c|}
\hline Frequency Range & $19102170 \mathrm{MHz}$ \\
\hline Gain & $17.2 \mathrm{dBi}$ \\
\hline Front to Back Ratio & more than $25 \mathrm{~dB}$ \\
\hline Electrical Down Tilt (RET) & 0-10 degrees \\
\hline
\end{tabular}

Table 2. : Antenna Specification

of the radio processing hardware. The main parts of the RRU are the followings:

- Transceiver (TRX)

- Transmitter (TX) amplification

- Transmitter/Receiver (TX/RX) duplex

- TX/RX filtering

- Voltage Standing Wave Ratio (VSWR) support

- Tower Mounted Amplifier (TMA) and Remote Electrical Tilt (RET) support.

-Optical interface

(3) Optical Interface Link The RRU are connected to the MU through optical fiber cables, where units can be connected to each other in several different ways depending on the site setup. The RBS can supports the followings:

- Star connection of the RRU, where each RRU is connected to the MU.

- RRUW and RRUS can support cascade connections, where only one fiber cable is connected between the MU and one of the RRU, while other RRUs are then connected to each others, this solution reduces the length of the optical fiber cable needed and can be used in multiple applications when the RRU are located far away from the MU.

(4) Antenna Configuration The antenna configuration recommended being able to configure different setups to cope with the strategic installation and keeping the existing infrastructure (co-located GSM and UMTS). All antenna configurations assume that the number of antenna ports available equals the number of feeder lines that can be installed. During the design we select antennas with specifications according to the project need and selected area as stated in table 2

4.1.3 Traffic Requirements. Considering both area and population requirements, the traffic requirements will vary depending on the area type (dense urban, urban, sub-urban, rural and road). Whereas the percentage of subscribers using each service is used to calculate "Average Subscriber Traffic Profile" as stated in the followings:

(1) Speech Traffic Requirements: The subscriber Busy Hour (BH) traffic profile for speech must be calculated from the given requirements in terms of:

-Busy Hour Call Attempts (BHCA).

- Call Mean Hold Time (MHT).

—Grade of Service $\backslash$ Blocking Probability (GOS $\left.\backslash P \_b\right)$. while, assuming the existence of 50,000 subscribers in $3 \mathrm{G}$ network which will use speech service, the average subscriber traffic profile is equal to: $(50,000 / 60,000) * 100 \%=83 \%$

(2) Speech Traffic Requirements calculation: By assuming that $\mathrm{BHCA}=0.5$ and $\mathrm{MHT}=3 \mathrm{~min}=0.05$ hours. Traffic (Nablus) $=$ BHCA X MHT. Traffic (Nablus) $=0.5$ X $0.05=0.025 \mathrm{E}=25$ $\mathrm{mE}$. Average Subscriber Speech Traffic $=25$ X 83\% $=20.75$ $\mathrm{mE}$.
(3) CS64 Traffic Requirements: The subscriber Busy Hour (BH) traffic profile for CS64 is to be calculated based on the following given requirements in terms of:

-Busy Hour Call Attempts (BHCA)

- Call Mean Hold Time (MHT).

—Grade of Service $\backslash$ Blocking Probability (GOS $\left.\backslash P \_b\right)$.

While, assuming that 10,000 subscribers to be exists in $3 \mathrm{G}$ network which will use CS64 service, the average subscriber traffic profile is to equal: $(10,000 / 60,000) * 100 \%=17 \%$

and by assuming that $\mathrm{BHCA}=0.1$, and $\mathrm{MHT}=6 \min (=0.1$ hours). Traffic (Nablus) $=$ BHCA X MHT. Traffic (Nablus) $=$ $0.1 \mathrm{X} 0.1=0.01 \mathrm{E}=10 \mathrm{mE}$. Average Subscriber Speech Traffic $=10 \times 17 \%=1.7 \mathrm{mE}$.

(4) PS Traffic Requirements: The term "PS Traffic" is used to distinguish between Third Generation Partnership Project (3GPP) Release 99 (R99 or UMTS) data services which have a maximum rate of $384 \mathrm{kbps}$ and High Speed data services that employs High Speed Downlink and Uplink Packet Access channels (HSDPA/HSUPA) [6]. The subscriber Busy Hour (BH) traffic profile for R99 PS have to be calculated from the given requirements in terms of:

-Uplink Traffic Volume [Kbyte $\backslash \mathrm{h}]$.

-Downlink Traffic Volume [Kbyte $\backslash \mathrm{h}]$.

when, assuming that 40,000 subscribers in $3 \mathrm{G}$ network will use PS R99 service, the average subscriber traffic profile is equal to:

$(40,000 / 60,000) * 100 \%=67 \%$

(5) R99 PS Traffic Requirements:

This requirement is already given in Kbyte $\backslash \mathrm{h}$, and we assumed that the uplink traffic is $10 \%$ of the downlink. Average Subscriber R99 DL PS Traffic $=60$ X 67\% $=40.2$ Kbyte $\backslash$ h. Average Subscriber R99 UL PS Traffic $=6$ X 67\% $=4.02$ Kbyte $\backslash \mathrm{h}$.

\subsection{Radio Network Dimensioning}

During the design, we use Ericsson Radio Network Proposal Tool (RNPT) to perform R99 dimensioning and preparing the Bill of Quantity (BoQ), which details the hardware required to implement the radio network design, this information can be used for pricing purpose. We can summarize this process into the following steps:

Step 1 Calculating the limitation on the capacity on basis of the maximum allowed traffic load on both directions; uplink and downlink.

Step 2 Based on the available given number of sites, compute and determine the actual cell load on both directions; uplink and downlink.

Step 3 Calculating the interface margin at uplink (B_IUL) and ensure it is greater than $0 \mathrm{~dB}$.

Step 4 Calculating the required power at the Common Pilot Channel $(\mathrm{CPICH})$ at the reference point $\left(\mathrm{C}_{-}(\mathrm{PICH}, \mathrm{ref})\right)$ take into consideration, that the power must be less than $10 \%$ of the nominal power at the reference point $\left(\mathrm{P}_{-}(\right.$nom,ref $)$).

Step 5 Calculating the total power at the reference point (P_(tot,ref)) and check that it must be less than $75 \%$ of nominal power at the reference point ( $\mathrm{P}_{-}($nom,ref) ).

Step 6 Calculating the required power at the maximum transport Dedicated Channel $(\mathrm{DCH})$ at the reference point $\left(\mathrm{P}_{-}(\mathrm{DCH}, \mathrm{ref})\right)$, where the calculated power must be ess than $30 \%$ of the nominal power at the reference point ( $\left.\mathrm{P}_{-}(\mathrm{nom}, \mathrm{ref})\right)$. 


\begin{tabular}{|c|c|c|}
\hline Service & Dense Urban & Subscribers using Service \\
\hline Speech BHCA & 0.5 & $83 \%$ \\
\hline Speech MHT & $3 \mathrm{~min}$ & $83 \%$ \\
\hline Speech Speech GOS & $2 \%$ & $83 \%$ \\
\hline CS64 BHCA & 0.1 & $17 \%$ \\
\hline CS64 MHT & $6 \mathrm{~min}$ & $17 \%$ \\
\hline CS64 GOS & $2 \%$ & $17 \%$ \\
\hline R99 PS UL Traffic [Kbyte $\backslash \mathrm{h}]$ & 6 & $67 \%$ \\
\hline R99 PS DL Traffic [Kbyte $\backslash \mathrm{h}]$ & 60 & $67 \%$ \\
\hline
\end{tabular}

Table 3. : Network Requirements

Table 3 states the network requirements for Nablus city, based on the output and findings from the R99 dimensioning process, where the maximum load should not exceed $70 \%$ and $76 \%$ uplink and downlink direction respectively.

\subsection{Radio Propagation Model Tuning:}

The 3rd Generation Partnership Project (3GPP [2]) and Third Generation Partnership Project 2 (3GPP2) industry alliances jointly developed channel models that are to be used for the evaluation of cellular systems with multiple antenna elements. The models are defined for three environments, namely urban microcells, urban macrocells, and suburban macrocells. The model is a mixed geometrical stochastic model that can simulate a cellular layout including interference, where as one of the most important steps is to tune the propagation model in order to get the best model parameters, that fit the land terrain to have the efficient coverage prediction, during the cell planning process, radio cell planning tool is used to predict the radio coverage by means of propagation models, for a particular site configuration. Different propagation models are considered according to the different environments and site configurations. The Algorithm 9999 model [18] is without knife-edge and spherical earth loss contribution implemented by Ericsson and based on the Okumura- Hata model [10] which is the best suited for large cell coverage (distances up to $100 \mathrm{~km}$ ) and it can extrapolate predictions up to the $2 \mathrm{GHz}$ band. This model has been proven to be accurate and is used by computer simulation tools. The earlier mentioned propagation model is the one adopted by Wataniya Mobile. The model tuning (model calibration) is performed in order to obtain more reliable radio propagation predictions. Measured and predicted signal strength samples are compared, and the mean error between them is minimized.

\subsection{Coverage and Nominal Cell Planning:}

The service footprint and availability is the most important key Performance Indicators (KPIs) in any wireless technology, this KPI determine if the end customer can access the network or not. In order to meet the design required coverage area, we have to classify and study the areas aspects i.e. population, building types, land terrain, $\ldots$ all the mentioned issues need advanced tools to automatically iterate the calculations.

\subsection{Site Search and Survey}

After designing the radio nominal points, the ideal scenario is to install the sites based on the exact location outcome from the planning tool, but in reality it usually it differs due to the geographical constraints, streets, houses, and some other environmental factors, thus site survey is to to be performed which translates the ideal solution to be an actual solution, and mostly we will shift or move the nominal points into near points due to ground obstacles i.e. leasing problem, nominal points locates in a middle of major street, not accessible piece of lands, no electricity, or any other arising constraints that may appear in site. Continuous refining and replanning tasks will be accomplished during the site survey to maintain the major coverage objectives.

\section{CONCLUSIONS}

The success of $3 \mathrm{G}$ mobile network depends on the efficient network planning, where the evolution from $2 \mathrm{G}$ to $3 \mathrm{G}$ networks is crucial nowadays, due to the different aspects and services offered by the $3 \mathrm{G}$ mobile network. This paper identify and analyze the relationship between capacity and coverage planning in addition to the concept of the quality of service.

This paper shows and discusses the methodology followed to perform the $3 \mathrm{G}$ network planning, including many different phases. Moreover, the design architecture take into consideration the traffic and service demand in the future.

\section{REFERENCES}

[1] Edoardo Amaldi, Antonio Capone, and Federico Malucelli. Radio planning and coverage optimization of $3 \mathrm{~g}$ cellular networks. Wireless Networks, 14(4):435-447, 2008.

[2] Prodip Chaudhury, Werner Mohr, and Seizo Onoe. The 3gpp proposal for imt-2000. Communications Magazine, IEEE, 37(12):72-81, 1999.

[3] Vijay Kumar Garg. Wireless Network Evolution: $2 G$ to $3 G$. Prentice Hall PTR, Upper Saddle River, NJ, USA, 1st edition, 2001.

[4] D. Goodman and R.A. Myers. 3g cellular standards and patents. In Wireless Networks, Communications and Mobile Computing, 2005 International Conference on, volume 1, pages 415-420 vol.1, June 2005.

[5] Liang Guo, Jie Zhang, and Carsten Maple. Coverage and capacity calculations for $3 \mathrm{~g}$ mobile network planning. proc. PGNET2003, June, pages 16-17, 2003.

[6] Harri Holma and Antti Toskala. HSDPA/HSUPA for UMTS: high speed radio access for mobile communications. John Wiley \& Sons, 2007.

[7] Patrick A Hosein. Qos control for wcdma high speed packet data. In Mobile and Wireless Communications Network, 2002. 4th International Workshop on, pages 169-173. IEEE, 2002. [8] TEMS Investigation. Tems investigation. WW.
ascom.com/nt/en/index-nt/tems-products-3/ tems-investigation-5.htm\#overview 2015. [Online; accessed 20-October-2015]

[9] Farooq Khan. LTE for 4G mobile broadband: air interface technologies and performance. Cambridge University Press, 2009.

[10] A. Medeisis and A. Kajackas. On the use of the universal okumura-hata propagation prediction model in rural areas. In Vehicular Technology Conference Proceedings, 2000. VTC 2000-Spring Tokyo. 2000 IEEE 51st, volume 3, pages 18151818 vol.3, 2000.

[11] A. Molisch. GSM Global System for Mobile Communications. Wiley-IEEE Press, 2011. 
[12] Wataniya Mobile powered by Blue Technology and Development. Wataniya mobile, member of ooredoo group. www. wataniya.ps/en/, 2016. [Online; accessed 10-January2016].

[13] Charalabos Skianis. Introducing automated procedures in $3 \mathrm{~g}$ network planning and optimization. Journal of Systems and Software, 86(6):1596-1602, 2013.

[14] Saed Tarapiah, Kahtan Aziz, and Shadi Atalla. Common radio resource management algorithms in heterogeneous wireless networks with kpi analysis. International Journal of $A d$ vanced Computer Science \& Applications, 1(6):53-58, 2015.

[15] Saed Tarapiah, Kahtan Aziz, and Shadi Atalla. Radio resource management in heterogeneous networks, functional models and implementation requirements. International Journal of Computer Applications, 127(16):1-4, 2015.

[16] Saed Tarapiah, Kahtan Aziz, Shadi Atalla, and Yousef Tarabeih. Advanced radio resource management solutions for multi-access wireless and mobile technologies. International Journal of Enhanced Research in Science, Technology \& Engineering (IJERSTE ), 4(9):165-169, 2015.

[17] Yufei Wu and Samuel Pierre. Optimization of $3 \mathrm{~g}$ radio network planning using tabu search doi: 10.14209/jcis. 2007.4. Journal of Communication and Information Systems, 22(1), 2015.

[18] S. Yelen, S. Selim Seker, and F. C. Kunter. Radio propagation path loss prediction of umts for an urban area. In Electromagnetic Field Computation (CEFC), 2010 14th Biennial IEEE Conference on, pages 1-1, May 2010. 